\title{
Minimally Invasive, Non-Thermal Tissue Ablation with a Single Exponential Decay Electrolytic Electroporation Waveform
}

\author{
Boris Rubinsky ${ }^{1,2}$, Enric Gunther ${ }^{1,2,3}$, Florin Botea ${ }^{4,5}$, Franco Lugnani ${ }^{6}$, Vlad Herlea ${ }^{5,7}$, Paul Mikus ${ }^{2}$, \\ Mihail Pautov $^{4,5}$, Nina Klein ${ }^{2,3}$, Catalin Pecheanu ${ }^{5,7}$, Michael K. Stehling ${ }^{1,2,3}$, Dana Tomescu ${ }^{5,8}$, \\ Matteo Macchioro ${ }^{6}$, Simona Dima ${ }^{4,5}$, Alexandru Serban ${ }^{9}$, Irinel Popescu ${ }^{4,5}$
}

${ }^{1}$ Department of Bioengineering and Department of Mechanical Engineering, University of California, Berkeley, USA

'Inter Science, Luzern, Switzerland

${ }^{3}$ Institut fuer Bildgebende Diagnostik, Offenbach, Germany

${ }^{4}$ Center of General Surgery and Liver Transplantation - Fundeni Clinical Institute, Bucharest,

Romania

${ }^{5}$ Center of Translational Medicine - Fundeni Clinical Institute, Bucharest, Romania

${ }^{6}$ Hippocrates D.0.0, Divaĉa, Slovenia; Clinica Santa Elena, Malaga, Spain

${ }^{7}$ Department of Pathology - Fundeni Clinical Institute, Bucharest, Romania

${ }^{8}$ Department of Anesthesiology and Intensive Care - Fundeni Clinical Institute, Bucharest,

Romania

${ }^{9}$ Department of Building Services, Transilvania University, Brasov, Romania
Corresponding author:

Boris Rubinsky

Professor of mechanical engineering Department of Bioengineering and Department of Mechanical Engineering University of California, Berkeley, CA 94720, USA

E-mail: brubinsky@gmail.com

\section{ABSTRACT}

A new minimally invasive tissue ablation technique, that combines the biophysical processes of electroporation and electrolysis, is introduced. The technology employs electrode needles inserted in the target tissue to deliver an Electrolytic Electroporation Waveform (EEW) in the form of an exponential decay voltage, several tens of microsecond long. A case study in a large animal model demonstrates that clinically significant size lesions can be achieved with a single, milliseconds long EEW. Ablation with EEW has major advantages over the comparable tissue ablation techniques of electrolysis, reversible electroporation and irreversible electroporation. EEW is orders of magnitude faster than conventional electrolytic ablation. EEW does not require the use of muscle relaxant as irreversible electroporation and EEW does not require injection of drugs as reversible electroporation. This new technology is simple to use and may become an important addition to the minimally invasive surgery armamentarium.

Key words: electroporation, electrolysis, tissue ablation technique, experimental surgery

\section{INTRODUCTION}

Minimally invasive surgery is a relatively recent branch of surgery, made possible by the advent of new technologies. An important application of
Received: 02.12 .2016

Accepted: 19.12 .2016

Copyright (c) Celsius Publishing House 
minimally invasive surgery is for tissue ablation. Various technologies are used for tissue ablation, including; radiofrequency, ultrasound, lasers, electrolytic ablation, photothermic ablation, freezing, electrochemotherapy, chemical ablation and more recently nonthermal irreversible electroporation. The various technologies have each their own advantages and disadvantages which dictate the specific applications for which they are used. In the past, most minimally invasive ablation technologies employed biophysical processes that ablate the entire mass of organic molecules in the targeted volume; such as thermal ablation with heat (1) or freezing (2) or chemical ablation with alcohols (3). These ablation technologies destroy the undesirable cells together with the connective tissue and vasculature and nerves in the treated volume.

Two technologies for minimally invasive tissue ablation, which selectively affect only some of the organic molecules in the treated volume leading to cell death, currently exist. They are electrolytic ablation and electroporation (reversible electroporation with chemotherapeutic drugs and irreversible electroporation). In electrolytic tissue ablation, a direct current flows between two electrodes bracing the targeted tissue. Cell death is caused by the chemical interaction between the products of electrolysis (in particular the changes in $\mathrm{pH}$ ) and cells $(4,5)$. A review of the use of electrolytic ablation of tumors in the liver and pancreas reports that "A specific advantage of electrolytic ablation is its safety even when conducted close to major vessels, while a disadvantage is the longer ablation times compared to more frequently employed techniques..... Pancreatic electrolytic ablation has been investigated in experimental studies that confirmed similar advantages to those found with liver ablation, but has never been evaluated on patients." (6). Indeed, electrolytic ablation times range from tens of minutes to hours. These long ablation times, in addition to problematic predictability due to diffusion and perfusion effects, are a major disadvantage of electrolytic ablation.

Electroporation is the permeabilization of the cell membrane achieved by exposing a cell to brief, nanosecond and microsecond long, high electric field pulses. Reversible electroporation results from certain electric pulses that reversible open the cell membrane. When certain toxic chemicals, such as bleomycin, enter the reversible electroporated cells, they cause cell death (7). Combining reversible electroporation with the injection of drugs ablates the tissue and spares the connective tissue and blood vessels. However, it requires the injection of a drug. The injection of the drug increases the complexity and uncertainty of the procedure.

Non-thermal irreversible electroporation is an ablation technology in which higher electric fields and numerous pulses are used to irreversible open the cell membrane, and, thereby induce cell death (8). This technology also spares the extracellular matrix and tissue structures like larger vessels and nerves, and is very effective in treating pancreatic cancer. However, the high electric fields cause muscle contractions and require the use of high doses of paralyzing drugs. Furthermore, the pulses are usually delivered at a frequency of about $1 \mathrm{~Hz}$. Therefore, while the pulses are microsecond long, a typical protocol that employs several hundred pulses could become many minutes long.

Recently, we have developed a new minimally invasive tissue ablation technology that combines electrolysis with electroporation, delivered as an Electrolytic Electroporation Waveform (EEW) (9-11).

Our earlier studies employed waveforms that delivered electrolysis and electroporation sequentially and separately. The results have shown that the combination of electrolysis and electroporation can ablate cells permeabilized by electroporation in a much shorter time than conventional electrolytic ablation. The studies of those combinations of electroporation and electrolysis have suggested that it should be possible to deliver the combination of electroporation and electrolysis simultaneously, using an exponential decay voltage waveform (EDW). A preliminary, acute study, in a small rodent animal model, has demonstrated the feasibility of tissue ablation with EDW. The results have shown that a very short, single millisecond EDW can ablate large volumes of tissue without attendant muscle contraction (12). We found that the EDW pulse combines the advantages of electrolytic ablation, reversible electroporation and irreversible electroporation, without the disadvantages. This report is a case study report, from a much larger study in which the effectiveness of EDW was investigated in a large animal (pig). The very promising results have motivated the publication of this first report from the forthcoming much larger study.

\section{MATERIALS AND METHODS}

Experiments were conducted in compliance with all ethical and legal rules imposed by national legislation and European Union. The experiment protocol was approved by the Ethics Committee of Fundeni Clinical Institute, and by the Bucharest Sanitary- 
Veterinary Authority (no 316).

The experimental study was carried out on an in vivo $80 \mathrm{~kg}$ Topigs 20 breed female pig. After being fasted for 24 hours, the animal was pre-medicated with a combination of acepromazine $(0.5 \mathrm{mg} / \mathrm{kg})$ and ketamine $(15 \mathrm{mg} / \mathrm{kg}$ ) injected intramuscularly (IM). Anesthesia was induced with intravenous (IV) Propofol $(2.5 \mathrm{mg} / \mathrm{kg}$ ) and $0.1 \mathrm{mg}$ Fentanyl. Endotracheal intubation was performed and anesthesia was maintained with sevoflurane in $80 \%$ oxygen (adjusted to $2-2.5 \%$ End-tidal sevoflurane). Possible postoperative pain was treated with morphine $0.1 \mathrm{mg} / \mathrm{kg} \mathrm{IM}$ and ketoprofen $1 \mathrm{mg} / \mathrm{kg}$ q 6 hours. Cefazolin $25 \mathrm{mg} / \mathrm{kg}$ IV was administrated every 2 hours.

The pig was placed in a ventral side-up position, and restrained using strings. The liver was exposed via an upper midline incision continued with a right transverse incision. The treatment was delivered using two 18gauge Titanium needles (Inter Science $\mathrm{GmbH}$, Switzerland) with a variable length (1-4 $\mathrm{cm}$ exposed treatment length) insulating sheath inserted in the liver, placed under ultrasound-guidance $(\mathrm{Hi}$ Vision Preirus Ultrasound device, Hitachi Medical Systems, Germany). Titanium was chosen, because, unlike steel or aluminum it is chemically inert, is biocompatible and at room temperature inert to oxygen, chloride and corrosion (13). In addition, $\mathrm{Ti}$ is MRI compatible. The 18-gauge variable length electrodes were custom designed for the delivery of both electroporation and electrolytic pulse sequences.

The experiment was carried out in an open-surgery setting to maximize the availability of liver lobes. The delivery of the single exponential decay waveform solely took place through the described needle-type electrodes. For each ablation lesion, two electrodes were inserted in the liver parenchyma under ultrasound monitoring, distant to large vessels (at least 1 $\mathrm{cm}$ ), in a roughly axial parallel configuration.

Since no apparatus is currently available to produce the exponential decay voltage waveform needed for the EEW procedure, we have designed and built a new power supply described in the following device section. This first large animal feasibility study was designed according to the $3 R$ principles for animal care (Replacement, Reduction, Refinement) of Russell and Burch (14). To this end, we have explored the effect of various EEW parameters in one pig liver. The pig liver was chosen because it allowed us to experiment with a large number of EEW parameters, while minimizing the number of animals used for the study. The pig liver from which this case study was extracted was treated with a total of 10 different lesions located in the left lateral lobe
(3 lesions), the left medial lobe (3 lesions), the right lateral lobe (2 lesions), and right medial lobe (2 lesions), respectively. Figure 1 depicts the intraoperative procedure during ablation for lesion number eight. It shows the way in which the two electrodes were inserted in the liver using a spacer. An important clinical aspect of all minimally invasive tissue ablation techniques is the need to ablate all the cells in the targeted tissue. Therefore, the focus of our investigation was to ascertain that EEW can completely ablate the tissue between the two electrode needles. Blood samples were taken pre-treatment, $1 \mathrm{~h}$ after treatment end time, $6 \mathrm{~h}$ after treatment end time, and before harvest to detect cytokines (sIL-2R, IL-10, IL-6), white blood cells and HGF. The surgery time was 65 minutes.

Postoperatively, the animal was kept alive for 24 hours, after which the liver was harvested and the animal was euthanized using $\mathrm{KCl} 7.45 \% 1 \mathrm{ml} / \mathrm{kg}$.

The liver harvesting procedure was carried out under general anesthesia. The surgical access was obtained by extending the midline incision. The liver hilum was dissected exposing the main bile duct, hepatic arteries and portal vein. The portal vein was catheterized via splenic vein, and the infrarenal aorta was dissected and cannulated through a direct approach. The descending aorta and the inferior vena cava were exposed intra-thoracically using a right frenotomy. The cross-clamping (clamping of the aorta in the thorax and above the iliac bifurcation, and of the intrathoracic inferior vena cava) was then performed, the inferior vena cava was sectioned below the liver, and the liver was flushed with $4 I$ of heparinized saline solution through the two cannulas at a hydrostatic pressure of $80 \mathrm{mmHg}$ from a pressurized IV drip.

The liver was then harvested and the flushing

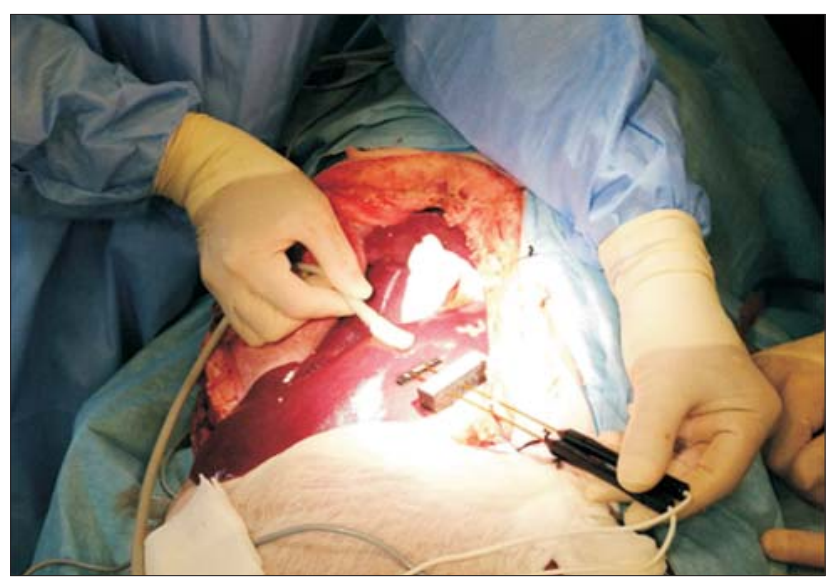

Figure 1 - Intraoperative aspect of the ablation procedure 
continued with the same solution until clean blood-free outflow from the hepatic veins was obtained. Immediately following saline perfusion, a $10 \%$ formalin fixative was perfused in the same way for ten minutes. All lesions were removed from the liver with normal surrounding parenchyma, and stored in the same formalin solution. For microscopic analysis, the tissue was bread loafed perpendicular to the capsule surface and parallel to the needle tracts. All cassettes were processed routinely from $10 \%$ phosphate buffered formalin (8-10 hours), processed routinely, and embedded in paraffin blocks. To examine the specimen histopathologically, three micrometer $(3 \mu \mathrm{m})$ sections were cut from each paraffin block, then hematoxylin \& eosin, and Masson's trichrome staining were performed for microscopic examination. The focus of the histology was to verify the extent and nature of tissue ablation with EDW. To produce information of practical clinical value, the focus of the analysis was on verifying the ability to produce a continuous lesion between the electrodes.

\section{DEVICE}

We were unable to find a power supply that can produce the waveform parameters, required for an EEW protocol in tissues with the clinical relevant dimensions, such as the pig liver. Therefore, we designed a power supply that operates in the modality of capacitor discharge electroporation systems (e.g. Gene Pulser Xcell ${ }^{\mathrm{TM}}$ Electroporation System, BioRad, Hercules, CA, USA) with an enhanced performance. The conventional type capacitors used were replaced with a bank of selectable capacitors allowing capacitance selection from $35 \mu \mathrm{F}$ to $218 \mathrm{uF}$ to provide the charge required for electrolysis. Similar to the Gene Pulser $\mathrm{Xcell}^{\mathrm{TM}}$, the generator has an output of up to $3 \mathrm{kV}$. Because of the larger capacitors, it can generate exponential decay waveforms up to time ranges of tens to hundred milliseconds, depending on tissue conductivity and thereby simultaneously deliver electrolysis and electroporation. The apparatus selects and matches the internal components needed to produce the time constants selected for the specific tissue conductivity of the treatment area by selecting an appropriate capacitor. The apparatus is able to produce and deliver the exponential decay voltage profile in the time and voltage range for the specific treatment area. Since capacitors carry a defined charge $\left(Q=C^{*} \cup 0\right)$, the amount of electrolytic species produced but also the Joule heating is predefined with the pre-settings of the total charge delivered.
The delivered pulses were logged using an oscilloscope coupled to a PC with a custom log- and statistical evaluation software written in Mathematica ${ }^{\circledR}$ (Wolfram Reseach, Mathematica ${ }^{\circledR}$ 10.0).

\section{RESULTS AND DISCUSSION}

The result presented in this case study was obtained by inserting two 18 gauge Ti needles separated by a distance of $15 \mathrm{~mm}$, in parallel, normal to the outer surface of the needle. The depth of insertion was 15 $\mathrm{mm}$ and the active treatment length was $15 \mathrm{~mm}$ from the tip. First, a brief $500 \mathrm{~V}$ pulse was delivered between the electrodes to verify the integrity of the electrical circuit. For the analyzed lesion (lesion no 6), induced on the diaphragmatic surface of the medial left lobe, this was followed by an exponential decay wave form with the following parameters: peak voltage $960 \mathrm{~V}$, capacitance charge - 122 pulse time constant $97 \mathrm{~ms}$. The parameters chosen for this study were drawn from the experimental results of (15).

They were chosen to deliver substantial amounts of electrolytic products. Minimal muscle contraction occurred, which did not necessitate the use of muscle relaxants.

The left hand side panel of figure 2 shows the trace of the voltage during the delivery of the EDW. The exponential nature of the shape is evident, though not perfectly exponential as secondary surface effects on the electrodes make resistance non-constant during pulse delivery. The right hand side panel of figure 2 shows the ultrasound appearance of the treated lesion immediately at the end of the delivery of the EDW. The needles signature is the two dark, hypoechoic, parallel lines. The most significant feature is the hyperechoic region. These are gases produced by electrolysis and evidence that electrolysis has occurred.

The focus of the histology was to verify the extent and nature of tissue ablation with EDW. To produce information of practical clinical value, the focus of the analysis was on verifying the ability to produce a continuous lesion between the electrodes.

Out of the ten ablated lesions that were pathologically analyzed, the most representative (lesion no 6) with low energy is depicted in figures 3 and 4 . Figure 3 shows a macroscopic cross section in a plane parallel to the direction of the needles. The sites from which the microscopic cross sections were taken are marked. Figure 4 depicts the microscopic appearance at those sites. The ablated liver area had a hemorrhagic appearance, with clear demarcation between ablated tissue and adjacent normal (transition zone) liver 


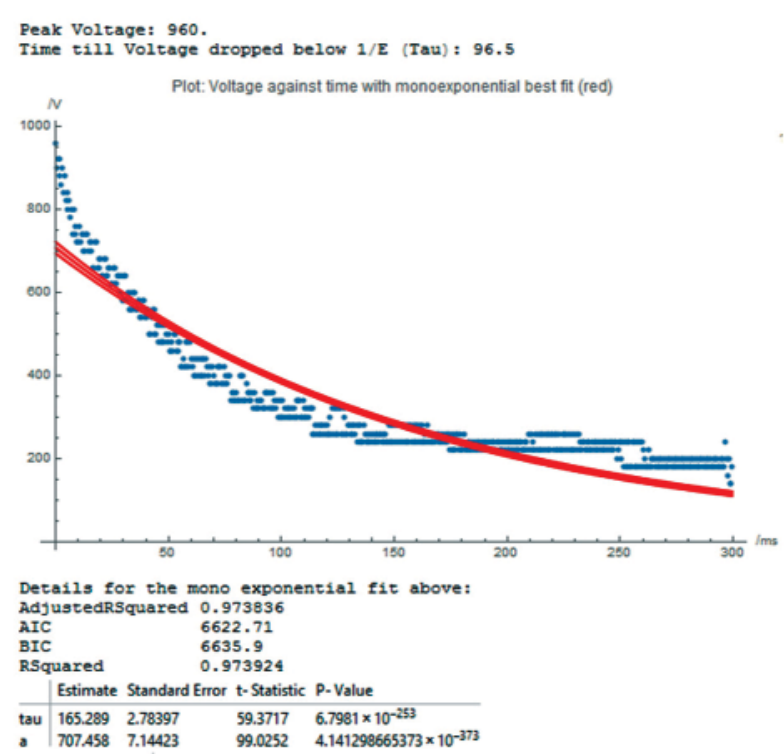

parenchyma; there was no fibrosis to delimitate these areas. The central area between electrodes had coagulative necrosis of liver parenchyma and hepatocytes with hyper eosinophilic cytoplasm and pyknotic nuclei. The sinusoids were congestive, filled with fibrin and inflammatory cells.

In some areas, in the central and peripheral areas congestion, edema and diffuse inflammatory cells (neutrophils, lymphocites, macrophages, and eosinophils) were detected. Inflammatory infiltrate and edema were present in the portal stroma. Outside the needles tracts, the parenchyma and large vessels were normal. The regional lymph nodes were also analyzed: they were of normal size and some of them with cortical reactivity, reactive germinal centers surrounding by lymphocytes (nonspecific reactive lymphadenitis). Thus, the ablation method succeeded in inducing necrosis between the two needles, while respecting the vessels outside these needles. To the best of our knowledge, this is the first reported successful tissue ablation with clinically relevant volume done in the fraction of a second.

\section{CONCLUSION}

This case study report has introduced a new minimally invasive tissue ablation technology that combines electrolysis and electroporation. Experiments in the pig liver show that clinical size ablation can be achieved with a single, milliseconds long, Electrolytic Electroporation Waveform (EEW), without the need for muscle paralysis as in the irreversible electroporation technology, or injection of drugs as in the

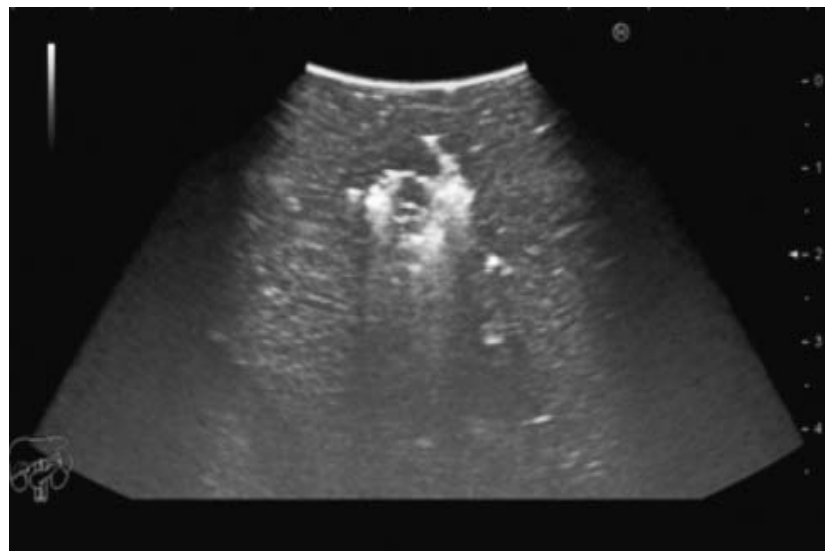

Figure 2 - Left panel shows the voltage trace as a function of time during the delivery of the EDW and statistical details for the delivered pulse and the best monoexponential fit parameters. Right panel shows the ultrasound image of the treated volume, immediately after the delivery of the EDW

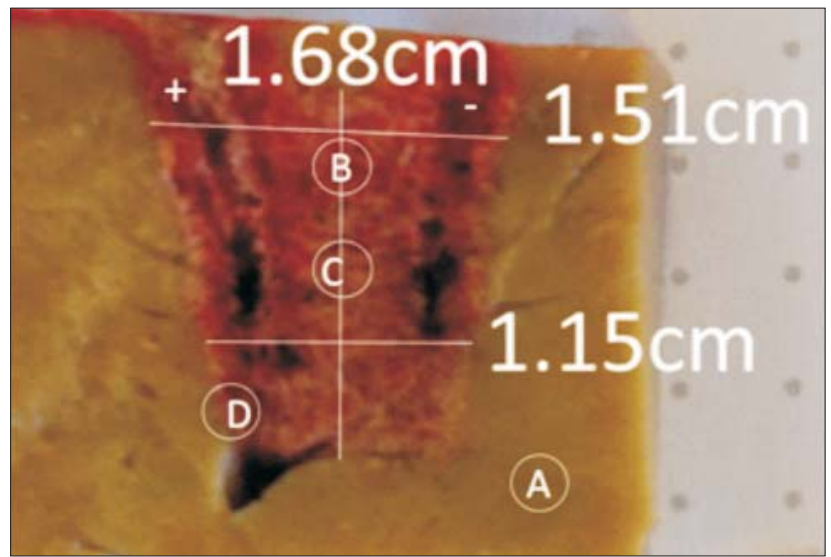

Figure 3 - Macroscopic gross section of the ablated area perpendicular to liver capsule surface and parallel to the needle tracts. The dimensions of the lesion are given in $\mathrm{cm}$. The sites from which samples for microscopy were taken are marked: $A$ - distant to the anode tract, as control; B - between the needle tracts, distant from the tips; $\mathbf{C}$ - between the needle tracts, close to the tips; D - transition zone outside the catode tract. The locations of the anode and cathode are also marked with (+) and (-)

reversible electroporation technology. The results suggest this technology may find important applications in the treatment of liver and pancreatic cancer.

\section{Acknowledgements}

We thank our colleagues from the Center of Translational Medicine - Fundeni Clinical Institute, Dr. Mihai Popescu (anesthesiologist), Dr. Radu Ionescu (veterinary), Anca Nastase (researcher), Andrei Sorop (researcher), who all provided insight and expertise that greatly assisted the research. 


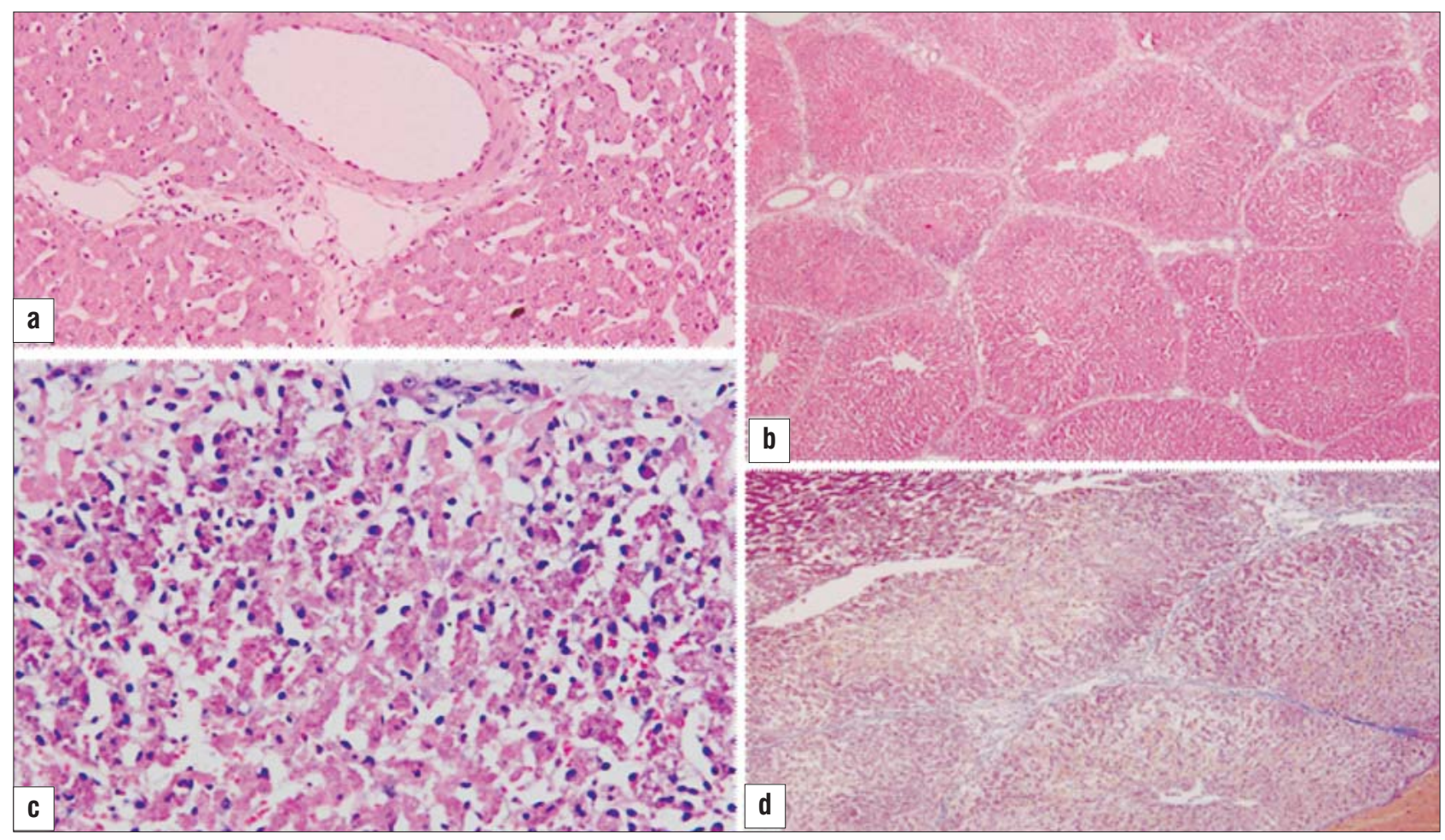

Figure 4 - Microscopical examination of the ablated lesion. a. location A - distant to the anode tract (normal liver parenchyma - control area): H\&E stained section (400x) - normal area of hepatic parenchyma and portal tract, for comparison to treated area. b. location B between the needle tracts, distant from the tips (target area): H\&E stained section (100x) hepatic parenchyma with hypereosinophilic cytoplasms, pyknotic nuclei and congestion of sinusoids; c. location C - between the needle tracts, close to the tips (target area): H\&E stained section (400x) - hepatic necrosis, hepatocytes with hypereosinophilic cytoplasms, pyknotic nuclei, congestion, edema and diffuse inflammatory cells. d. location D - transition area outside the catode tract: Masson trichrome stained section (100x) - hepatic parenchyma with hypereosinophilic cytoplasms, pyknotic nuclei and congestion of sinusoids

\section{REFERENCE}

1. Tiong L, Maddern GJ. Systematic review and meta-analysis of survival and disease recurrence after radiofrequency ablation for hepatocellular carcinoma. Br J Surg. 2011 Sep;98(9):1210-24. doi: 10.1002/bjs. 7669. Epub $2011 \mathrm{Jul} 15$.

2. Rubinsky B Cryosurgery. Annu Rev Biomed Eng. 2000;2:157-87.

3. Dodd GD 3rd, Soulen MC, Kane RA, Livraghi T, Lees WR, Yamashita Y, et al. Minimally invasive treatment of malignant hepatic tumors: at the threshold of a major breakthrough. Radiographics. 2000 Jan-Feb; 20(1):9-27.

4. Nilsson E, von Euler H, Berendson J, Thörne A, Wersäll P, Näslund I, et al. Electrochemical treatment of tumours. Bioelectrochemistry. 2000 Feb;51(1):1-11

5. Czymek R, Dinter D, Löffler S, Gebhard M, Laubert T, Lubienski A, et al. Electrochemical treatment: An investigation of dose-response relationships using an isolated liver perfusion model. Saudi J Gastroenterol. 2011 Sep-0ct;17(5):335-42. doi: 10.4103/13193767.84491.

6. Gravante G, Ong SL, Metcalfe MS, Bhardwaj N, Maddern GJ, Lloyd $\mathrm{DM}$, et al. Experimental application of electrolysis in the treatment of liver and pancreatic tumours: principles, preclinical and clinical observations and future perspectives. Surg Oncol. 2011 Jun;20(2):106-20. doi: 10.1016/j.suronc.2009.12.002. Epub 2010 Jan 4.

7. Mir LM, Belehradek M, Domenge C, Orlowski S, Poddevin B Belehradek JJ, Schwaab G, Luboinski B, Paoletti C. Electrochemotherapy, a new antitumor treatment: first clinical trial. Comptes
Rendus de l'Academie des Sciences Serie III Sciences de la Vie, 313. 1991, p. 613-618.

8. Rubinsky B, Onik G, Mikus P. Irreversible electroporation: a new ablation modality--clinical implications. Technol Cancer Res Treat. 2007 Feb;6(1):37-48.

9. Phillips M, Rubinsky L, Meir A, Raju N, Rubinsky B. Combining Electrolysis and Electroporation for Tissue Ablation. Technol Cancer Res Treat. 2015 Aug;14(4):395-410. doi: 10.1177/ 1533034614560102. Epub 2014 Nov 21.

10. Phillips M, Raju N, Rubinsky L, Rubinsky B. Modulating electrolytic tissue ablation with reversible electroporation pulses. Technology. 2015;3:45-53.

11. Stehling MK, Guenther E, Mikus P, Klein N, Rubinsky L, Rubinsky B. Synergistic Combination of Electrolysis and Electroporation for Tissue Ablation. PLoS One. 2016 Feb 11;11(2):e0148317. doi: 10.1371/journal.pone.0148317. eCollection 2016.

12. Phillips M, Krishnan H, Raju N, Rubinsky B. Tissue Ablation by a Synergistic Combination of Electroporation and Electrolysis Delivered by a Single Pulse. Ann Biomed Eng. 2016 0ct;44(10):3144-54. doi: 10.1007/s10439-016-1624-4. Epub 2016 May 4.

13. Emsley J. "Titanium". Nature's Building Blocks: An A-Z Guide to the Elements. Oxford University Press, Oxford, England, UK, 2001.

14. Russell WMS, Burch RL. The principles of humane experimental technique, The principles of humane experimental technique, DOI (1959) $x i v+238 p-x i v+238 p$.

15. Phillips M, Krishnan H, Raju N, Rubinsky B. Tissue ablation by a synergistic combination of electroporation and electrolysis delivered by a single pulse. Annals of Biomedical Engineering. 2016;44(10): 3144-3154 DOI DOI: 10.1007 /s10439-016-1624-4. 